\title{
Therapieoptimierung bei Knochenmetastasen
}

Hintergrund und Fragestellung: Knochenmetastasen können zur Destruktion von Knochengewebe und diversen Komplikationen führen. Mit Einführung der Bisphosphonate und später von Denosumab (DM) gelang es, die ossäre Morbidität signifikant zu mindern. Dabei sprachen einige Studienergebnisse dafür, dass DM skelettbezogene Ereignisse im Vergleich zu Zoledronsäure (ZS) wirksamer verhindert. Die subkutane Applikation von DM erleichtert zudem die chronische Anwendung. Darüber hinaus ist der Einsatz von $\mathrm{ZS}$ bei Patienten mit Niereninsuffizienz aufgrund der Nephrotoxizität eingeschränkt. Fengxia Chen und Feifei Pu betrachteten nun im Rahmen einer Metaanalyse Wirksamkeit und Sicherheit beider Substanzen im direkten Vergleich [1].

Patienten und Methodik: Für die Metaanalyse wurden Daten von PubMed, EMBASE, Cochrane Library, Web of Science mit den Conference Proceedings,

Originalie

Chen F, Pu F. Safety of Denosumab Versus Zoledronic Acid in Patients with Bone Metastases: A Meta-Analysis of Randomized Controlled Trials. Oncol Res Treat. 2016;39(7-8):453-9. Elsevier und die China National Knowledge Infrastructure (CNKI) durchsucht. Zwei unabhängige Gutachter extrahierten die Daten aus jeder geeigneten Studie. Die Daten von sechs prospektiven, kontrollierten Studien mit insgesamt 13.733 Patien- ten wurden analysiert und evaluiert. Dafür wurden sowohl „Random-“ als auch „Fix-Effects-Modelle“ genutzt.

Ergebnisse: Das Auftreten einiger unerwünschter Arzneimittelwirkungen (uAW) unter DM (120 mg subkutan, q4w) und ZS (4 mg intravenös, $\mathrm{q} 4 \mathrm{w}$ ) war bei Patienten mit Knochenmetastasen relativ vergleichbar, so z. B. Nausea, Fatigue, Asthenie, Arthralgien und Knochenschmerzen. Dagegen traten unter DM seltener Anämie (Odds Ratio [OR] 0,86, $95 \%$-Konfidenzintervall [95\%-KI] 0,79-0,94), Anorexie (OR 0,90, $95 \%-K I 0,82-0,98$ ) und Nephrotoxizität (OR 0,74, $95 \%$-KI 0,64-0,85) auf, grenzwertig seltener auch Rückenschmerzen (OR 0,94, $95 \%$-KI 0,86-1,03) und Obstipation (OR 0,89; $95 \%$-KI 0,77-1,04). UAW wie Hypokalzämie (OR 2,17, $95 \%$-KI 1,84-2,56) und die Entwicklung eines weiteren malignen Prozesses (OR 1,65, 95\%-KI 1,06-2,59) waren unter ZS seltener. Auch Kieferosteonekrosen fanden sich etwas seltener unter ZS (OR 1,29, 95\%-KI 0,95-1,76).

Schlussfolgerungen der Autoren: In dieser Metaanalyse erwies sich DM bei Patienten mit Knochenmetastasen als sicherer bezüglich einer Verschiebung bzw. Vermeidung skelettbezogener Ereignisse. Zudem verzögerte DM die Zunahme von Knochenschmerzen stärker als ZS.

Schlüsselwörter: Denosumab - Zoledronsäure - skelettbezogene Ereignisse - Kiefernekrose - Reboundeffekt

\section{- Kommentar von Ulrich R. Kleeberg, Hamburg}

\section{"Vor- und Nachteile müssen sorgsam abgewogen werden"}

Der zur Behandlung der Osteoporose und von Knochenmetastasen zugelassene RANKL-Antagonist Denosumab (DM; Prolia ${ }^{\circledR}$ mit 60 mg und Xgeva ${ }^{\circledR}$ mit 120 mg) bewirkt eine stärkere Hemmung der Knochenresorption und des Knochenumbaus. Zudem beugt er skelettbezogenen Ereignissen (SRE) besser vor als Bisphosphonate [2]. Nach Absetzen geht der Effekt jedoch wieder verloren: Rasch nimmt im Sinne eines Reboundeffekts die Knochendichte $\mathrm{ab}$ und die Marker des Knochenumbaus steigen auf Werte oberhalb der Ausgangswerte [3]. In diesem Zusammenhang können bei der Osteoporose unter DM spontan multiple schmerzhafte Wirbelfrakturen auftreten, die unter Bisphosphonaten so nicht beschrieben wurden. In einer Übersicht fassten Wolfgang Becker-Brüser und Kollegen eine Reihe unerwünschter Arzneimittelwirkungen zusammen [3], die angenommene Vorteile von DM im Vergleich zu Bisphosphonaten relativieren. Mehr dazu wurde in der hier vorgestellten Arbeit von Fengxia Chen und Feifei Pu zusammengetragen. Soll eine bestehende Therapie beendet werden, empfiehlt die Firma Amgen (Schweiz) eine sorgfältige Nachkontrolle der Osteoporosepatienten. Der begrenzten Datenlage zufolge kann eine Umstellung auf "eine andere antiresorptive Therapie (z.B. Bisphosphonate)" den Knochenmineralverlust mindern [3].
In der Onkologie gilt also ein sorgsames Abwägen der Vorund Nachteile - speziell im Hinblick auf patientengegebene Risiken. Zudem ist besondere Vorsicht geboten beim Einsatz von DM als adjuvante Maßnahme, z.B. um der Entwicklung einer Osteoporose aufgrund einer Therapie mit Aromataseinhibitoren oder einer Aussaat von Tumorzellen vorzubeugen.
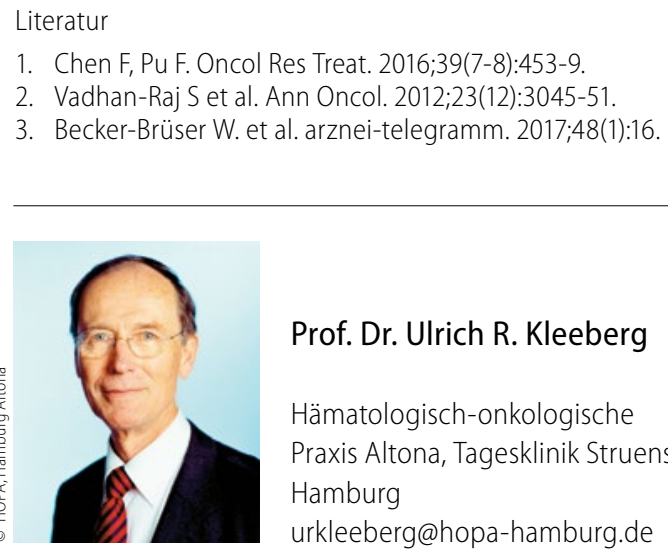

Prof. Dr. Ulrich R. Kleeberg Hämatologisch-onkologische Praxis Altona, Tagesklinik Struensee-Haus, Hamburg urkleeberg@hopa-hamburg.de 